\title{
The stellar content, metallicity and ionization structure of HII regions
}

\author{
N. L. Martín-Hernández ${ }^{1}$, R. Vermeij ${ }^{1}$, A. G. G. M. Tielens ${ }^{1,2}$, J. M. van der Hulst ${ }^{1}$, and E. Peeters ${ }^{2,1}$ \\ 1 Kapteyn Institute, PO Box 800, 9700 AV Groningen, The Netherlands \\ 2 SRON, National Institute for Space Research, PO Box 800, 9700 AV Groningen, The Netherlands
}

Received 22 October 2001 / Accepted 5 April 2002

\begin{abstract}
Observations of infrared fine-structure lines provide direct information on the metallicity and ionization structure of $\mathrm{H}$ II regions and indirectly on the hardness of the radiation field ionizing these nebulae. We have analyzed a sample of Galactic and Magellanic Cloud H II regions observed by the Infrared Space Observatory (ISO) to examine the interplay between stellar content, metallicity and the ionization structure of Hin regions. The observed [S IV] 10.5/[S III] $18.7 \mu \mathrm{m}$ and [Ne III] 15.5/[Ne II] $12.8 \mu \mathrm{m}$ line ratios are shown to be highly correlated over more than two orders of magnitude. We have compared the observed line ratios to the results of photoionization models using different stellar energy distributions. The derived characteristics of the ionizing star depend critically on the adopted stellar model as well as the (stellar) metallicity. We have compared the stellar effective temperatures derived from these model studies for a few well-studied H II regions with published direct spectroscopic determinations of the spectral type of the ionizing stars. This comparison supports our interpretation that stellar and nebular metallicity influences the observed infrared ionic line ratios. We can explain the observed increase in degree of ionization, as traced by the $[\mathrm{S} \mathrm{IV}] /[\mathrm{S} \mathrm{III}]$ and [Ne III]/[NeII] line ratios, by the hardening of the radiation field due to the decrease of metallicity. The implications of our results for the determination of the ages of starbursts in starburst galaxies are assessed.
\end{abstract}

Key words. stars: atmospheres - stars: early-type - ISM: abundances - ISM: H II regions - galaxies: starburst galaxies: individual: Milky Way - Magellanic Clouds

\section{Introduction}

The spectral type of the ionizing stars and the stellar and nebular metallicity are intimately interwoven in controlling the ionization structure of $\mathrm{HII}$ regions. Hotter stars will ionize trace elements to higher ionization stages. Metallicity, on the other hand, modifies the spectral energy distribution (SED) of the ionizing stars and thus, influences the ionization structure indirectly. In particular, line blocking/blanketing is directly coupled to the metallicity and controls the stellar wind of massive stars. The stellar wind, in its turn, also modifies the SED of the star. The interlocking of these different effects hampers the determination of the stellar content from ionic line

\footnotetext{
Send offprint requests to: N. L. Martín-Hernández, e-mail: leticia@astro.rug.nl

* Based on observations with ISO, an ESA project with instruments funded by ESA Member States (especially the PI countries: France, Germany, The Netherlands and the UK) and with the participation of ISAS and NASA.
}

observations. This has clear repercussions on the study of starburst regions in galaxies as well, where the stellar content is used as an indicator of the starburst age. Current models of stellar atmospheres have started to take the effects of metallicity into account (e.g. Schaerer \& de Koter 1997; Pauldrach et al. 2001). Such models can fit the observed line ratios in $\mathrm{H}$ II regions reasonable well (e.g. Giveon et al. 2001; Morisset et al. 2002). The derived spectral types for the ionizing sources, however, depend on the stellar model adopted and can be very different.

The Infrared Space Observatory (ISO, Kessler et al. 1996) has provided a powerful tool for the study of the ionization structure of $\mathrm{H}$ II regions (Giveon et al. 2001; Martín-Hernández et al. 2002, hereafter MHP02). The ratios of fine-structure lines originating in the ionized gas (for instance, [Ar III] 9.0/[Ar II $] 7.0 \mu \mathrm{m}$, [S IV] 10.5/[S III] $18.7 \mu \mathrm{m}$ and [Ne III] 15.5/[Ne II] $12.8 \mu \mathrm{m})$ are widely used to constrain the properties of the ionizing stars in these nebula (e.g. Watarai et al. 1998; Takahashi et al. 2000; Morisset et al. 2002). The systematic studies of the ionization structure of $\mathrm{H}$ II regions via these ionic line 
Table 1. Selected Hil regions in the Milky Way, Small Magellanic Cloud (SMC) and Large Magellanic Cloud (LMC). Given are the object name, the distance in kpc of the Galactic objects from the center of the Galaxy and the coordinates of the ISO pointing.

\begin{tabular}{|c|c|c|c|}
\hline Object & $\begin{array}{l}\text { Rgal } \\
(\mathrm{kpc})\end{array}$ & $\begin{array}{l}\mathrm{RA}(\mathrm{J} 2000.0) \\
\left(\mathrm{h},{ }^{\mathrm{m}},{ }^{\mathrm{s}}\right)\end{array}$ & $\begin{array}{c}\delta(\mathrm{J} 2000.0) \\
\left({ }^{\circ},{ }^{\prime},{ }^{\prime \prime}\right)\end{array}$ \\
\hline \multicolumn{4}{|l|}{ Milky Way: } \\
\hline IR $01045+6506$ & 13.8 & 010750.7 & +652121.7 \\
\hline IR $02219+6125$ & 11.0 & 022544.6 & +620611.3 \\
\hline IR $10589-6034$ & 9.5 & 110059.8 & -605027.1 \\
\hline IR $11143-6113$ & 9.7 & 111633.8 & -61 2959.4 \\
\hline IR $12063-6259$ & 9.3 & 120901.1 & -631554.7 \\
\hline IR $12073-6233$ & 10.1 & 121000.3 & -624956.5 \\
\hline IR $12331-6134$ & 6.9 & 123601.9 & -615103.9 \\
\hline IR $15384-5348$ & 6.4 & $15 \quad 42 \quad 17.1$ & -535831.5 \\
\hline IR $15502-5302$ & 4.6 & 155406.0 & -531136.4 \\
\hline IR $17221-3619$ & 5.2 & 172531.7 & -362153.5 \\
\hline IR $17455-2800$ & 0.5 & 174841.5 & -280138.3 \\
\hline IR $18116-1646$ & 4.3 & 181435.2 & -164520.6 \\
\hline IR $18317-0757$ & 4.5 & 183424.9 & -075447.9 \\
\hline IR $18434-0242$ & 4.6 & 184604.0 & -023920.5 \\
\hline IR $18479-0005$ & 7.5 & 185030.8 & -000159.4 \\
\hline IR $18502+0051$ & 4.7 & 185250.2 & +005527.6 \\
\hline IR $19598+3324$ & 9.8 & 200145.6 & +333243.7 \\
\hline Dr 21 & 8.6 & 203900.9 & +421941.9 \\
\hline IR $21190+5140$ & 12.7 & 212044.9 & +515326.5 \\
\hline IR $23030+5958$ & 11.4 & 230510.6 & +601440.6 \\
\hline \multicolumn{4}{|l|}{ SMC: } \\
\hline N66 & & 005903.7 & $-72 \quad 1039.9$ \\
\hline N81 & & 010913.6 & -731141.1 \\
\hline \multicolumn{4}{|l|}{ LMC: } \\
\hline $\mathrm{N} 4 \mathrm{~A}$ & & 045208.4 & -665523.4 \\
\hline N83B & & 045425.2 & $-69 \quad 1059.8$ \\
\hline N11A & & 045716.2 & -662318.3 \\
\hline 30 Dor\#1 & & 053833.5 & -690627.1 \\
\hline 30 Dor\#2 & & 053835.5 & -690541.2 \\
\hline 30 Dor\#3 & & 053846.0 & -690507.9 \\
\hline 30 Dor\#4 & & 053854.2 & -690515.3 \\
\hline N160A1 & & 053943.3 & -693851.4 \\
\hline N160A2 & & 053946.1 & -693836.6 \\
\hline N159-5 & & 054002.4 & -694433.4 \\
\hline
\end{tabular}

ratios as a function of Galactic center distance in the Milky Way by Martín-Hernández et al. (2002) and in the Large and Small Magellanic Clouds by Vermeij \& van der Hulst (2002) have now provided us with a "natural" sample of $\mathrm{H}$ II regions over a wide range in metallicity. Such a sample allows us to study the effect of stellar type and metallicity on the ionization structure. Moreover, while the ionizing stars in most $\mathrm{H}$ II regions are heavily extincted in the visible by tens to hundreds of magnitudes, the opening up of the infrared window has favoured in recent years the use of infrared spectra to infer the spectral types of the ionizing stars. An important effort has now started to identify the ionizing stars of Galactic H II regions in the $K$-band
(Hanson et al. 1996; Watson \& Hanson 1997) and the first results are now becoming available (Kaper et al. 2002a,b).

In this paper, we examine this interplay between stellar content, metallicity and ionization structure of $\mathrm{HII}$ regions based upon the ISO sample. This paper is structured as follows. Section 2 describes the combined sample of Galactic and LMC/SMC H II regions; Sect. 3 shows the correlation between the observed [NeIII] $/[\mathrm{NeII}]$ and [S IV]/[S III] line ratios. Sections 4 and 5 investigate the influence of the SEDs and the stellar/nebular metallicity, respectively, on the ionization structure. Section 6 discusses the implications for the interpretation of the spectra of starburst galaxies. Finally, Sect. 7 discusses and summarizes the results.

\section{The sample}

We combined the ISO Short Wavelength Spectrometer (de Graauw et al. 1996) observations of the Galactic and Magellanic Cloud H II regions as described in the catalogues by Peeters et al. (2002) and Vermeij et al. (2002). These catalogues present the atomic fine-structure line fluxes of 43 Galactic nebulae and 12 Magellanic Cloud sources. The first analysis of the observed fine-structure lines is presented in MHP02 and Vermeij \& van der Hulst (2002). This analysis includes, among others, the derivation of elemental abundances and a discussion on the ionization state of the nebulae.

From this combined set of $\mathrm{H}$ II regions, we selected those for which both information on their elemental abundances (through $\mathrm{Ne} / \mathrm{H}$ ) and ionization state (through the [S IV] 10.5/[S III] $18.7 \mu \mathrm{m}$ and [Ne III] 15.5/[Ne II $12.8 \mu \mathrm{m}$ line ratios) could be derived. The selected H II regions are listed in Table 1, where the source name, the distance of the Galactic objects from the center of the Galaxy and the ISO pointing coordinates are given. We chose to use $\mathrm{Ne} / \mathrm{H}$ as a tracer of the nebular metallicity. $\mathrm{Ne} / \mathrm{H}$ can be determined from the IR fine-structure lines to higher precision than other elements because it is less sensitive to ionization correction factors and assumptions regarding electron densities (see MHP02 for a complete discussion). Moreover, as a primary element, the abundance of neon closely follows that of oxygen (e.g. Henry \& Worthey 1999, and references therein). As far as the choice of the ionization tracers is concerned, the choice of the $[\mathrm{S} \mathrm{IV}] /[\mathrm{S} \mathrm{III}]$ and $[\mathrm{Ne} \mathrm{III}] /[\mathrm{Ne} \mathrm{II}]$ line ratios was an obvious one as $[\mathrm{Ar} \mathrm{III}] /[\mathrm{Ar} \mathrm{II}]$ has been detected in only a few LMC H II regions.

In total, our sample consists of 20 Galactic H II regions located at Galactocentric distances between 0 and $15 \mathrm{kpc}$, $7 \mathrm{HII}$ regions in the LMC (including 4 positions in 30 Doradus) and 2 in the SMC. Also included are the SWS observations of $\mathrm{Sgr} \mathrm{A}^{\star}$, our own Galactic Center (Lutz et al. 1996), and the Orion nebula, for which an "integrated" infrared spectrum was obtained by Simpson et al. (1998) using the Midcourse Space Experiment (MSX) satellite. The MSX field of view $\left(6^{\prime} \times 9^{\prime}\right)$ included most of the Orion nebula. 


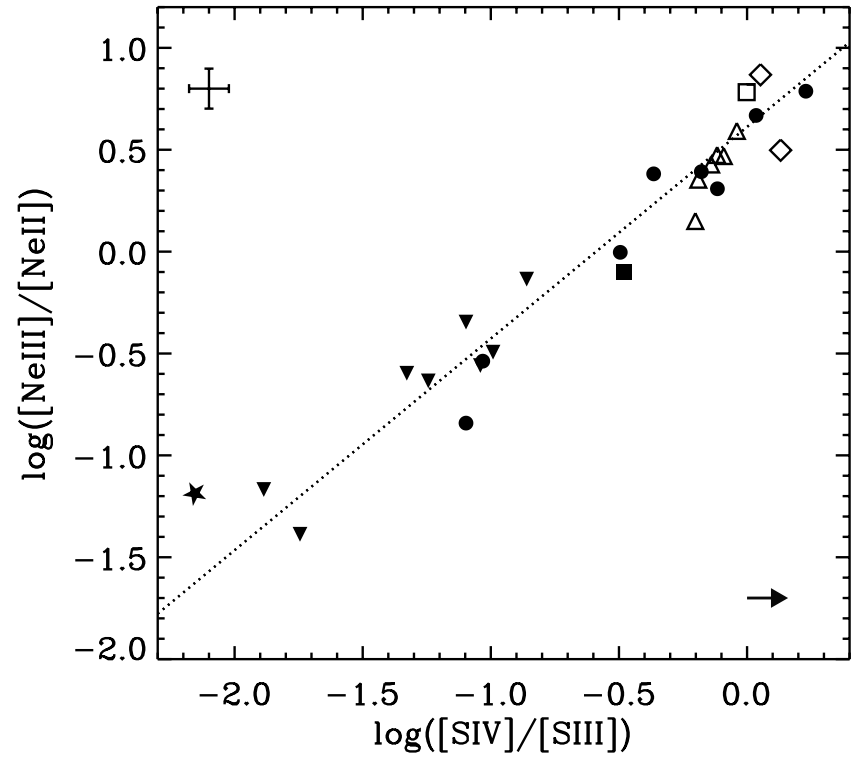

\section{Variations in the ionization structure}

The ionization structure of a nebula depends basically on the shape of the SED and the nebular geometry. In the case of an ionized sphere of constant gas density and filling factor, the geometrical effect can be defined by the ionization parameter $U=Q_{\mathrm{H}} /\left(4 \pi R^{2} n c\right)$, where $Q_{\mathrm{H}}$ is the number of stellar photons above $13.6 \mathrm{eV}$ emitted per second, $R$ is the Strömgren radius, $n$ is the gas density and $c$ is the speed of light. The ionization structure can be traced by the ratio of successive stages of ionization $X^{+i}$ and $X^{+i+1}$ of a given element. Such a ratio depends, for a given $U$, on the number of photons able to ionize $X^{+i}$ (Vílchez \& Pagel 1988).

In Fig. 1, we present the relation between the $[\mathrm{S} \mathrm{IV}] /[\mathrm{S} \mathrm{III}]$ and the $[\mathrm{Ne} \mathrm{III}] /[\mathrm{NeII}]$ line ratios for the combined sample of $\mathrm{H}$ II regions in the Milky Way and the LMC/SMC. The line ratios have not been corrected for extinction. Because the lines [Ne II] 12.8 and [Ne III] $15.5 \mu \mathrm{m}$ are very close in wavelength, the $[\mathrm{Ne} \mathrm{III}] /[\mathrm{Ne} \mathrm{II}]$ is practically insensitive to extinction. However, the differential extinction between the [S IV] 10.5 and [S III] $18.7 \mu \mathrm{m}$ lines ( $\sim 0.08 A_{K}$ if a standard extinction curve is considered, see MHP02) can affect the $[\mathrm{S} \mathrm{IV}] /[\mathrm{S}$ III] line ratio. The effect of this differential extinction is indicated in the lower right corner of Fig. 1 for a typical $A_{K}$ of 2 mag (MHP02).

The data shown in Fig. 1 span a range in ionization of more than 2 orders of magnitude. The LMC/SMC points nicely overlap the Galactic trend at the high ionization end. The least squares fit to the data, displayed as a dotted line, gives a slope of $1.04 \pm 0.05$ and an intercept of $0.61 \pm 0.04$. Figure 1 also illustrates the change in the degree of ionization of the Galactic $\mathrm{H}$ II regions with increasing distance from the Galactic center. The more highly ionized objects are located at larger Galactocentric distances, while the less highly ionized objects, including $\mathrm{Sgr} \mathrm{A}^{*}$, are located at smaller distances. In line with the known metallicity gradient in the Galaxy (e.g. see review
Fig. 1. Relationship between the $[\mathrm{S}$ IV $] 10.5 /[\mathrm{S} \mathrm{III}] 18.7 \mu \mathrm{m}$ and [Ne III] $15.5 /[\mathrm{Ne}$ II] $12.8 \mu \mathrm{m}$ line ratios for the combined sample of $\mathrm{H}$ II regions. Indicated by various symbols are the Galactic nebulae at $R_{\text {Gal }}<7 \mathrm{kpc}$ (solid triangles), the Galactic nebulae at $R_{\text {Gal }}>7 \mathrm{kpc}$ (solid circles), the LMC nebulae (open triangles, except 30 Doradus, which is indicated by an open square) and the SMC nebulae (open diamonds). To avoid confusion, only the averaged location of the 4 SWS positions in 30 Doradus is indicated. The positions of $\mathrm{Sgr} \mathrm{A}{ }^{*}$ (Lutz et al. 1996) and the Orion nebula (Simpson et al. 1998) are indicated by a solid star and a solid square, respectively. The dotted line is a least squares fit to the data. A typical error bar of the ISO data is given in the upper left corner. The arrow in the lower right corner indicates the extinction correction due to a $A_{K}=2$ mag.

by Henry \& Worthey 1999; Rolleston et al. 2000, and references therein), the location of the high metallicity Galactic Center at the low ionization end of the correlation and the low metallicity LMC/SMC H II regions at the high ionization end suggests that metallicity is somehow involved in the changes of ionization degree, and hence, in the hardening of the radiation field, shown in Fig. 1.

We consider in the next sections both the influence of the SED and the metallicity on the ionization structure of $\mathrm{H}$ II regions.

\section{Influence of the SEDs}

In order to investigate the dependence of the [Ne III]/[Ne II] and $[\mathrm{S} \mathrm{IV}] /[\mathrm{S} \mathrm{III}]$ line ratios on the SED, a set of nebular models has been calculated with the photoionization code CLOUDY (Ferland et al. 1998) using MICE, the IDL interface for CLOUDY created by H. Spoon ${ }^{1}$. The parameters varied in the modelling are the stellar effective temperature $\left(T_{\text {eff }}\right)$ and the ionization parameter $(U)$. We compute nebular models for a static, spherically symmetric, homogeneous gas distribution with one ionizing star in the center. An inner cavity with a radius equal to $10^{17} \mathrm{~cm}$ is set, while the outer radius of the HII region is defined by the position where the electron temperature, $T_{\mathrm{e}}$, reaches $100 \mathrm{~K}$. Two different stellar atmosphere models are taken to describe the SED: the CoStar models by Schaerer \& de Koter (1997) and the models by Pauldrach et al. (2001). Previous studies of these ionization ratios have concentrated on either the CoStar models (e.g. Morisset et al. 2002, MHP02) or on the Pauldrach models (Giveon et al. 2001). For a detailed study of the influence of these and other stellar SEDs on the ionization ratios, we refer to the work by Morisset et al. (in prep.). We use stellar models for main sequence (dwarf) stars, i.e. CoStar models A2, B2, C2, D2, E2 and F2,

1 See http://www . astro.rug.nl/ spoon/mice.html 


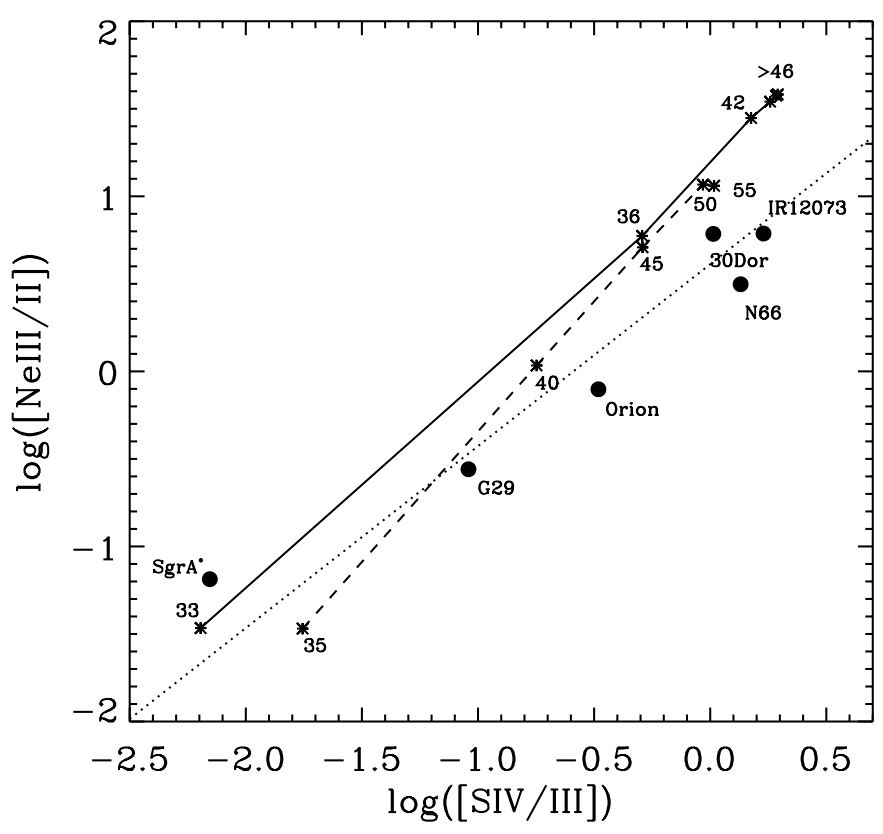

Fig. 2. Dependence of the $[\mathrm{S} I V] /[\mathrm{S} \mathrm{III}]$ and $[\mathrm{Ne} \mathrm{III}] /[\mathrm{Ne} \mathrm{II}]$ line ratios on the stellar $T_{\text {eff }}$ (crosses) as given by the CoStar (solid lines) and Pauldrach (dashed lines) SEDs. Only photoionization models for $n_{\mathrm{H}}=10^{3} \mathrm{~cm}^{-3}$ are plotted. The observed line ratios for reference sources (Sgr A*, G29.96-0.02, the Orion nebula, IRAS 12073-6233, N66 and 30 Dor) are indicated by solid circles. The dotted line is the least squares fit to the data plotted in Fig 1.

and Pauldrach models D-35, D-40, D-45, D-50 and D-55. The metallicity of the star and the nebula is chosen to be solar. The number of hydrogen ionizing photons emitted by the central source is fixed to the typical value of $Q_{\mathrm{H}}=10^{48}$ photons s $^{-1}$. The parameter $U$ is varied by changing the hydrogen density $\left(n_{\mathrm{H}}\right)$. For each of the stellar atmosphere models selected, which span a range in $T_{\text {eff }}$ from approximately 30 to $55 \mathrm{kK}$, photoionization models are calculated for $n_{\mathrm{H}}=10^{2}, 5 \times 10^{2}, 10^{3}, 5 \times 10^{3}$ and $10^{4} \mathrm{~cm}^{-3}$.

The output of this grid of models for $n_{\mathrm{H}}=$ $10^{3} \mathrm{~cm}^{-3}$ is presented in Fig. 2. The variation of $[\mathrm{NeIII}] /[\mathrm{NeII}]$ and $[\mathrm{SIV}] /[\mathrm{SIII}]$ with $T_{\text {eff }}$ is shown separately for the two selected SED models. An increase in density of two orders of magnitude produces an increase of the line ratios of at most 0.5 dex approximately along the lines drawn in Fig. 2. For reference, the observed line ratios of the Galactic sources Sgr A*, G29.96-0.02 (IRAS 18434-0242), the Orion nebula and IRAS 12073-6233, the SMC H II region N66, and 30 Doradus are indicated by solid circles. Sgr A* and IRAS 12073 delimit the observed parameter space for $[\mathrm{NeIII}] /[\mathrm{NeII}]$ and $[\mathrm{SIV}] /[\mathrm{SIII}]$ as they are, respectively, the lowest and highest ionized source in Fig. 1.

Several points can be gleaned from inspection of Fig. 2: (a) The $[\mathrm{Ne}$ III $] /[\mathrm{Ne}$ II $]$ and $[\mathrm{S} \mathrm{IV}] /[\mathrm{S} \mathrm{III}]$ line ratios strongly depend on the stellar $T_{\text {eff }}$, but they can no longer discriminate between models when most of the Ne and $\mathrm{S}$ is in the higher ionization stage (see also Giveon et al. 2001,

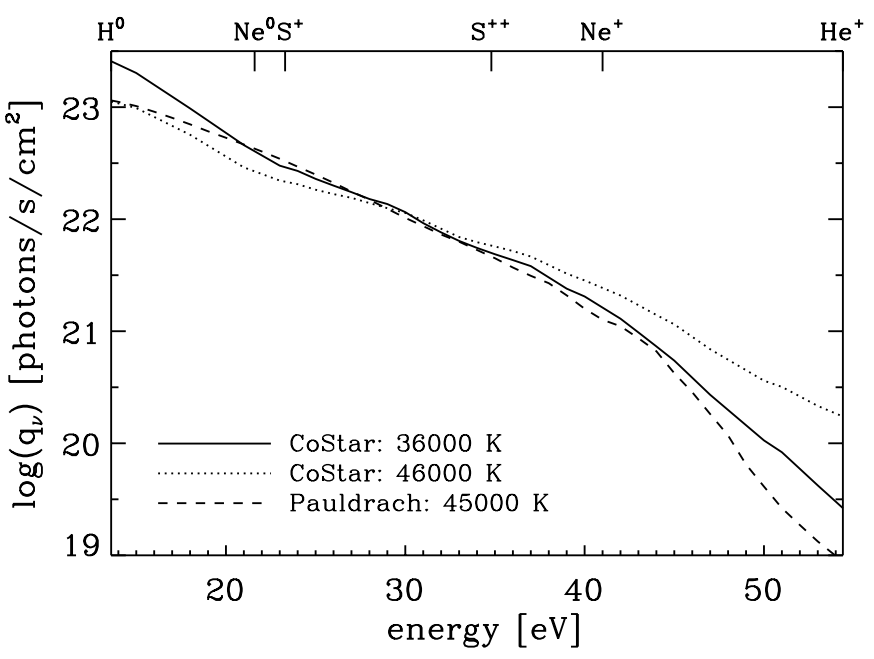

Fig. 3. The number of ionizing photons in $\mathrm{cm}^{-2} \mathrm{~s}^{-1}$ above a certain energy from the CoStar B2 $(36000 \mathrm{~K})$, D2 $(46000 \mathrm{~K})$ and Pauldrach D-45 $(45000 \mathrm{~K})$ SED models is plotted as a function of this energy. The curves are normalized so that the total number of hydrogen ionizing photons, $Q_{\mathrm{H}}$, is $10^{48}$ photons $\mathrm{s}^{-1}$, the value used in the photoionization models. Labeled on top of the figure are the ionization energies for $\mathrm{H}^{0}, \mathrm{Ne}^{0}, \mathrm{~S}^{+}, \mathrm{S}^{++}, \mathrm{Ne}^{+}$and $\mathrm{He}^{+}$.

MHP02). When this happens, these line ratios remain constant even with large changes in the SED. When using the CoStar SEDs, both [Ne III]/[Ne II] and [S IV] /[S III] become insensitive to the stellar $T_{\text {eff }}$ for $T_{\text {eff }}>42 \mathrm{kK}$, while when using the Pauldrach SEDs, the hardness of the stellar ionizing flux changes less rapidly with $T_{\text {eff }}$, and it is not until $T_{\text {eff }}>50 \mathrm{kK}$ that the star fully ionizes $\mathrm{S}^{++}$and $\mathrm{Ne}^{+}$.

(b) The slope of the observed $\log ([\mathrm{Ne} I \mathrm{II}] /[\mathrm{Ne} I \mathrm{I}])$ versus $\log ([\mathrm{S} \mathrm{IV}] /[\mathrm{S} \mathrm{III}])$ is practically equal to 1 (cf., Fig. 1), suggesting that the spectral hardening affects equally the range of ionizing energies for $\mathrm{S}$ III $(h \nu>35 \mathrm{eV})$ and Ne II $(h \nu>41 \mathrm{eV})$. The model trends are, however, slightly steeper than the data, i.e. for a given $T_{\text {eff }}$ the models overpredict $[\mathrm{Ne} \mathrm{III}] /[\mathrm{NeII}]$ (underpredict $[\mathrm{S} \mathrm{IV}] /[\mathrm{S} \mathrm{III}]$ ) by a factor up to 5 for a given $[\mathrm{S} \mathrm{IV}] /[\mathrm{S} \mathrm{III}]([\mathrm{Ne} \mathrm{III}] /[\mathrm{Ne} \mathrm{II}])$. As has been discussed by Oey et al. (2000) and Morisset et al. (2002), there is an indication that the CoStar models overpredict the stellar spectrum at these ionizing energies. (c) When using the CoStar SEDs to describe the emergent flux, practically the entire observed ionization range spanning more than 2 orders of magnitude can be modeled by a rather narrow $T_{\text {eff }}$ range between $\sim 33 \mathrm{kK}$ and $\sim 42 \mathrm{kK}$. This effect is less dramatic with the Pauldrach SEDs, for which the entire range up to $55 \mathrm{kK}$ is suitable for modelling the observed line ratios.

We see that large differences appear when we compare the predictions by the CoStar models to those by the Pauldrach ones for a given $T_{\text {eff }}$. These differences are primarily caused by the different treatment of the line blocking and blanketing between the two SED models. This causes the Pauldrach models to have a much softer 
Table 2. Comparison of the stellar properties predicted via the [NeIII]/[Ne II] and [S IV]/[S III] line ratios with the spectral type derived directly from infrared/optical stellar spectra. Given are the object name, the observed spectral type of the ionizing star(s) with its estimated $T_{\text {eff }}$, the predicted stellar properties by using either the CoStar or the Pauldrach SEDs and the reference of the observed spectral type. The objects are sorted by increasing degree of ionization.

\begin{tabular}{|c|c|c|c|c|c|c|}
\hline \multirow[b]{2}{*}{ Object name } & \multirow[b]{2}{*}{ Spectral Type } & \multicolumn{2}{|c|}{ Observed $T_{\text {eff }}^{\dagger}$} & \multicolumn{2}{|c|}{ Predicted $T_{\text {eff }}^{\dagger}$} & \multirow[b]{2}{*}{ Ref. } \\
\hline & & (a) & (b) & CoStar & Pauldrach & \\
\hline G 29.96-0.02 & O5-O3 & $46-51$ & $42-49$ & $33-36^{\star}$ & $35-40$ & 1 \\
\hline Orion nebula & $\mathrm{O} 6$ & 44 & 39 & $33-36$ & $40-45$ & 2 \\
\hline N66 & O6.5 and earlier & $>42$ & $>37$ & $33-42$ & $>40$ & 3 \\
\hline 30 Doradus & $\mathrm{O} 4-\mathrm{O} 3$ & $49-51$ & $43-49$ & $36-42$ & $>45$ & 4,5 \\
\hline IRAS 12073-6233 & $\mathrm{O} 7.5-\mathrm{O} 6$ & $40-44$ & $36-39$ & $>36$ & $>45$ & 1 \\
\hline
\end{tabular}

(a) $T_{\text {eff }}$ derived from the observed spectral type using the calibration by Vacca et al. (1996); (b) $T_{\text {eff }}$ derived from the observed spectral type using the calibration by Martins et al. $(2002) ;\left(^{\dagger}\right) T_{\text {eff }}$ in $\mathrm{kK} ;\left(^{\star}\right)$ The detailed photoionization model by Morisset et al. (2002), which uses the CoStar SEDs, predicts a $T_{\text {eff }}$ of $\sim 30_{-1}^{+2} \mathrm{kK}$. References: (1) Kaper et al. (2002a,b) and private communication; (2) SIMBAD database; (3) Massey et al. (1989); (4) Walborn \& Blades (1997); (5) Massey \& Hunter (1998).

spectrum than the analogous CoStar star at a similar $T_{\text {eff }}$. Note, for instance, the case of the CoStar SED with $T_{\text {eff }}=36000 \mathrm{~K}$ (model B2) and the Pauldrach SED with $T_{\text {eff }}=45000 \mathrm{~K}$ (model D-45) in Fig. 2, which predict practically the same values for $[\mathrm{S} \mathrm{IV}] /[\mathrm{S} \mathrm{III}]$ and $[\mathrm{Ne} \mathrm{III}] /[\mathrm{Ne} \mathrm{II}]$.

It is instructive to calculate the integrated ionizing photon flux above a certain energy as a function of this energy for these two models (CoStar B2 and Pauldrach $\mathrm{D}-45)$. The number of ionizing photons in $\mathrm{cm}^{-2} \mathrm{~s}^{-1}$ with energies larger than $h \nu_{i}$ is defined as $q_{\nu_{i}}=\int_{\nu_{i}}^{\infty} F_{\nu} /(h \nu) \mathrm{d} \nu$, with $F_{\nu}$ being the astrophysical flux in $\operatorname{erg~s}^{-1} \mathrm{~cm}^{-2} \mathrm{~Hz}^{-1}$. The results are shown in Fig. 3. For a significant comparison with the photoionization model results, the total number of hydrogen ionizing photons has been scaled to $10^{48}$ photons $\mathrm{s}^{-1}$, the value used in the models. We note that models differ in their stellar radii. The $[\mathrm{S}$ IV $] /[\mathrm{S}$ III $]$ and $[\mathrm{Ne} \mathrm{III}] /[\mathrm{Ne} \mathrm{II}]$ line ratios are basically sensitive to the slope of the curve between the energies able to produce and ionize $\mathrm{S}^{++}$and $\mathrm{Ne}^{+}$, i.e. between 23.3 and $34.8 \mathrm{eV}$, and 21.6 and $41.0 \mathrm{eV}$, respectively. The shapes of the CoStar B2 and the Pauldrach D-45 SEDs are very similar between these energies; hence, their match in the predicted $[\mathrm{S} \mathrm{IV}] /[\mathrm{S} \mathrm{III}]$ and $[\mathrm{Ne} \mathrm{III}] /[\mathrm{Ne} \mathrm{II}]$ line ratios. The ionization structure of a nebula with such values of $[\mathrm{S} \mathrm{IV}] /[\mathrm{S} \mathrm{III}]$ and $[\mathrm{Ne}$ III $] /[\mathrm{Ne}$ II $]$ can, therefore, be equally well described by any of these two SEDs. However, the interpretation of this ionization structure in terms of the stellar properties, e.g. $T_{\text {eff }}$, depends on the stellar model adopted. For comparison, we also show the shape of the hotter CoStar model D2, with a $T_{\text {eff }}$ of $46000 \mathrm{~K}$. The ionizing flux of this hotter CoStar model starts to be higher than the corresponding Pauldrach model above $\sim 30 \mathrm{eV}$. This hotter CoStar model will produce, consequently, a larger amount of $\mathrm{S}^{+3}$ and $\mathrm{Ne}^{++}$.

In this respect, closely related to the problem of determining the $T_{\text {eff }}$ of the ionizing sources is that of their spectral type classification. It is illustrative to consider at this point the cases of G29.96-0.02, the Orion nebula, IRAS 12073-6233, N66 and 30 Doradus, for which the spectral types of the ionizing stars are known. The Orion nebula is ionized by the Trapezium cluster, the dominant source of ionizing radiation being the $\mathrm{O} 6$ star $\theta^{1} \mathrm{C}$ Ori. 30 Dor is known to be powered by a stellar cluster containing a large number of O3-O4 stars (Walborn \& Blades 1997; Massey \& Hunter 1998). N66 is the most prominent $\mathrm{H}$ II region in the SMC. It contains a rich stellar cluster of at least $33 \mathrm{O}$ stars of which 11 are of type O6.5 or earlier (Massey et al. 1989). Recent high resolution $K$-band spectral observations of G29 and IRAS 12073 (Kaper et al. $2002 \mathrm{a}$,b, private communication) allow the determination of the spectral type of the ionizing stars based mainly upon the presence and strength of photospherical lines of C IV and N III (Hanson et al. 1996). These observations indicate that IRAS 12073 is ionized by a cluster of young O6-O7.5 stars, while the spectral type of the main ionizing star in G29 is confirmed to be O3-O5 (we note that lower resolution $K$-band spectral observations by Watson \& Hanson 1997 classified this star as being O5-O8).

As mentioned above, the interpretation of the $[\mathrm{SIV}] /[\mathrm{SIII}]$ and $[\mathrm{NeIII}] /[\mathrm{NeII}]$ line ratios observed for these objects in terms of the stellar $T_{\text {eff }}$ depends on the stellar model used to describe the ionization structure traced by these ratios. The subsequent spectral classification of the ionizing source depends on the spectral type attributed to the SED used in the modelling of the $\mathrm{H}$ II region. We can try to compare the $T_{\text {eff }}$ determined from the observed line ratios by using either CoStar or Pauldrach SEDS (cf., Fig. 2) with the observed spectral type of the ionizing star(s). To do so, we need a $T_{\text {eff }}$-spectral type calibration for $\mathrm{O}$ stars. The $T_{\text {eff }}$ calibration of the different spectral types is based on modelling the observed photospheric hydrogen, helium and metal lines and it is by no means certain. For instance, the commonly used

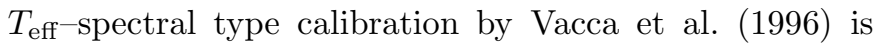
based on plane-parallel models which do not incorporate stellar winds and line blocking/blanketing. The more recent calibration by Martins et al. (2002), based on nonLTE line blanketed atmosphere models with stellar winds 


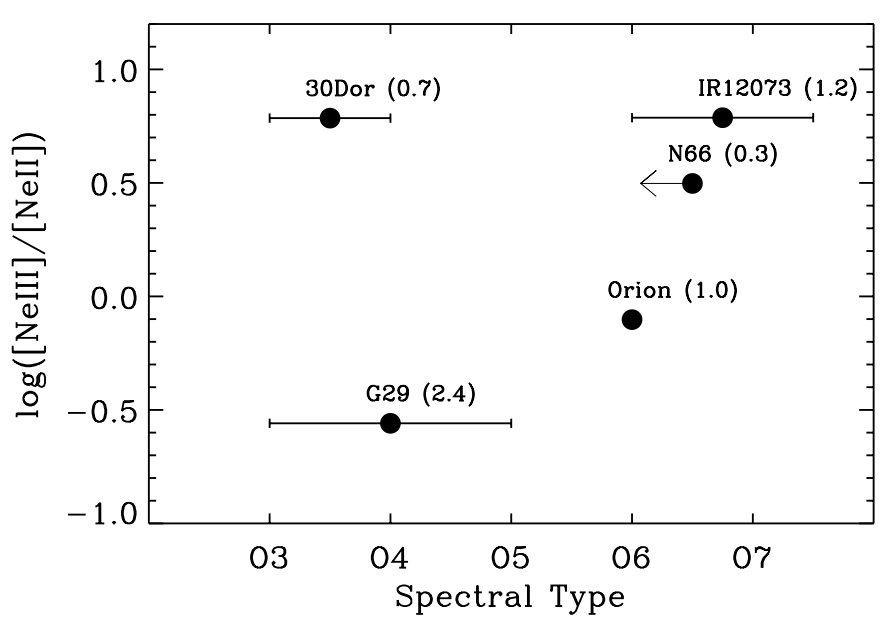

Fig. 4. The line ratio $[\mathrm{NeIII}] /[\mathrm{NeII}]$ is compared to the observed spectral type for G29.96-0.02, the Orion nebula, IRAS 12073-6233, N66 and 30 Doradus. The numbers in brackets indicate the $\mathrm{Ne} / \mathrm{H}$ abundance by number in units of $10^{-4}$. References for the $\mathrm{Ne} / \mathrm{H}$ abundances are: G29 and IRAS 12073 (MHP02); N66 and 30 Dor (Vermeij \& van der Hulst 2002); Orion (Simpson et al. 1998).

computed with the CMFGEN code of Hillier \& Miller (1998), gives substantially lower effective temperatures than the Vacca et al. (1996) calibration for the same spectral type. We will use both calibrations.

The result of the comparison between the observed spectral types and the predicted stellar properties is summarized in Table 2. The calibration by Martins et al. (2002) gives effective temperatures for the observed spectral types $\sim 4000-5000 \mathrm{~K}$ lower than Vacca et al. (1996). Even considering this most recent calibration, the CoStar models systematically predict a lower temperature for the ionizing stars than observed. A better match is found by using the Pauldrach SEDs, although the predicted $T_{\text {eff }}$ for G29 is still low. The opposite is true for IRAS 12073, for which the Pauldrach models predict a hotter star than observed. Moreover, while our photoionization models predict that an increase in the degree of ionization is coupled to an increase in $T_{\text {eff }}$ and thus in spectral type, observationally this trend is less clear (cf., Fig. 4). In particular, G29 and 30 Dor are both ionized by very early O stars, yet their ionization ratios are very different. Similarly, IRAS 12073 and 30 Dor are ionized by stars with very different spectral types but their ionization ratios are very similar. It is clear that the stellar spectral type as determined from optical or infrared spectroscopy is not the only controlling factor of the ionization structure of $\mathrm{H}$ II regions. In line with the discussion in Sect. 3 (cf., Fig. 1), we infer that the metallicity of the star and $\mathrm{HII}$ region is involved as well. At this respect, the differences in spectral type between G29 and 30 Dor, and in ionization structure between 30 Dor and IRAS 12073 may be a consequence of the differences in metallicity. G29 has a Ne/H abundance more than 3 times higher than 30 Dor; 30 Dor, on the other hand, has a lower abundance than IRAS 12073 (cf., Fig. 4).
It is well known that a stellar wind modifies the SED of a star. This effect is directly coupled to the strength of the wind, which in turn depends on the metallicity. Metallicity also controls the degree of attenuation of the UV and EUV stellar radiation due to line blocking and blanketing. Changes in metallicity can, therefore, transform the stellar spectrum to that of a cooler or hotter star than what is determined from the solar metallicity stellar models.

\section{Influence of the metallicity}

The ionization tracer $[\mathrm{Ne} \mathrm{III}] /[\mathrm{Ne} \mathrm{II}]$ is compared with the metallicity tracer $\mathrm{Ne} / \mathrm{H}$ in Fig. 5. The data show a loose trend of decreasing degree of ionization with metallicity. No selection effect is thought to influence this relationship. While some of the LMC/SMC H II regions are among the brightest in these galaxies - 30 Dor (LMC) and N66 (SMC) are powered by luminous stellar clusters containing many early $\mathrm{O}$ stars -, the LMC sample also contains sources such as N11A, which are alike to the H II regions in the Galactic sample in terms of luminosity and Lyman continuum flux. Besides, no selection effect is apparent in the Galactic H II regions, for which no obvious variation in the density of the ionized gas or the stellar luminosity with Galactocentric distance is observed (cf., MHP02). There is some systematic effect, however, due to the influence of metallicity on the electron temperature of the ionized gas. The Galactic Ne/H abundances were derived using $\operatorname{Br} \alpha$ to determine the $\mathrm{H}^{+}$emission associated to the nebula and a constant $T_{\mathrm{e}}$ of $7500 \mathrm{~K}$ was assumed. Hence, a high metallicity nebula, which is expected to have a lower electron temperature, will actually have a higher Br $\alpha$ emission coefficient than the one adopted in the evaluation of the $\mathrm{Ne} / \mathrm{H}$ abundance, and vice versa. For reasonable electron temperatures, between 5000 and $10000 \mathrm{~K}$ (Shaver et al. 1983; Afflerbach et al. 1996; Deharveng et al. 2000), this effect is less than a factor of 2 (cf., MHP02). The Magellanic Cloud abundances, however, are not expected to be influenced by this systematic effect because they were evaluated using electron temperatures derived from optical lines. Hence, we consider that the trend in Fig. 5 is not due to a selection effect, and that there is a general correlation albeit with a large spread. On average, the most highly ionized H II regions have the lowest metallicity, and vice versa.

Giveon et al. (2001) investigated the influence of varying the stellar and nebular metallicity on the [Ne III] $/[\mathrm{Ne} \mathrm{II}]$ line ratio based upon a set of photoionization models computed using the Pauldrach SEDs. They found that, for a given $T_{\text {eff }}$, the SED hardens with decreasing metallicity due to the decreased line blocking in the stellar atmosphere winds. Quantitatively, for a $40 \mathrm{kK}$ star, an increase in the nebular and stellar metallicity from $0.5 Z_{\odot}$ to $2 Z_{\odot}$ would reduce [Ne III] / $[\mathrm{Ne}$ II] by a factor of 20 . We compare the results of these calculations to the observed correlation between metallicity and degree of ionization (cf., Fig. 5) for $T_{\text {eff }}=35,40,45 \mathrm{kK}, Z=0.5 Z_{\odot}, 1 Z_{\odot}, 2 Z_{\odot}$ and 


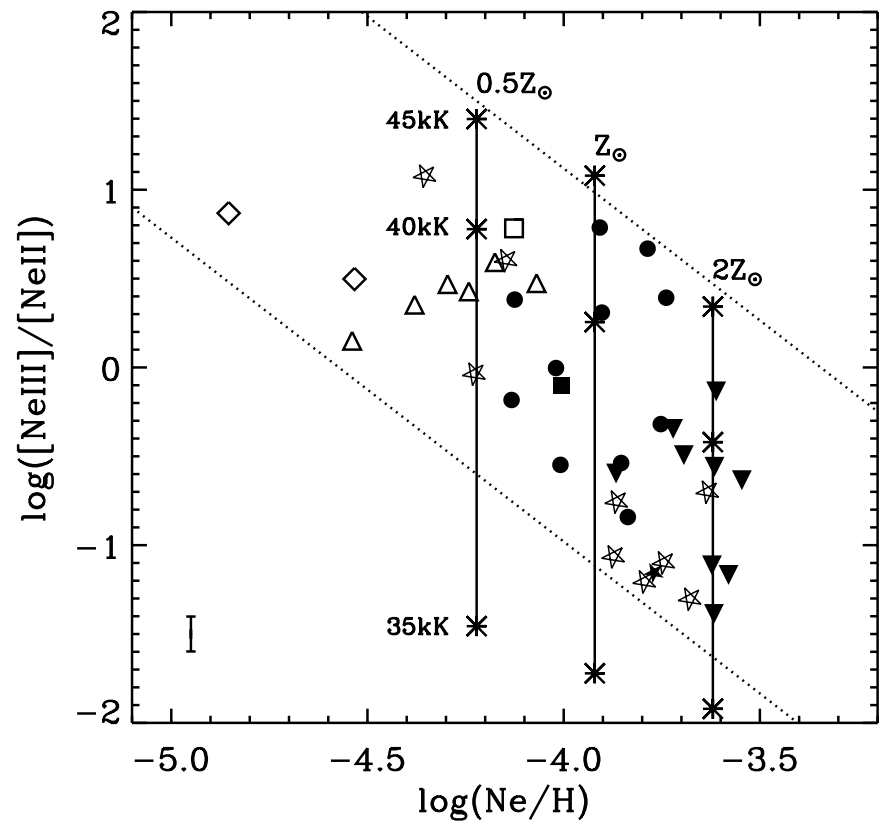

$n_{\mathrm{H}}=800 \mathrm{~cm}^{-3}$. In view of these results, we see that the large spread in degree of ionization at a given metallicity observed in our data reflects a true variation in $T_{\text {eff }}$. Moreover, the observed increase in the degree of ionization of the H II regions (cf., Fig. 1) reflects clearly a hardening of the SEDs due to the decreased metallicity and it is not necessarily due to a rise of the effective temperature of the ionizing star.

Returning to the individual sources considered in Sect. 4 and in view of Fig. 5, the Galactic H II region G29, with a metallicity of roughly $2 Z_{\odot}$, would be powered by a star with a temperature slightly higher than the one indicated in Fig. 2, which are solar metallicity models. The Magellanic sources N66 and 30 Dor, with sub-solar metallicities, would be, on the other hand, ionized by cooler stars because of the decreased line blocking. However, a proper comparison of the effective temperature derived from the modelling of the $\mathrm{H}$ II region line data with the effective temperature derived directly from the stellar spectral will require a proper calibration of stellar spectra for different metallicities. In particular, the "calibration" of ionizing stars based upon $K$-band spectra (Hanson et al. 1996) may require spectra of stars with different metallicities. Also, the comparison of the strength of hydrogen and helium lines with predictions of stellar atmospheres will require a proper treatment of the influence of metallicity.

\section{The case of starburst galaxies}

Information on starburst galaxies has been collected in order to make a comparison with the $\mathrm{H}$ II region results. The data obtained for a sample of 9 galaxies observed by ISO are presented in Table 3 and are plotted in Fig. 5 as open stars. These galaxies show a similar distribution as the H II regions: high metallicity-low ionization, low metallicityhigh ionization. In particular, we note that the low metallicity dwarf irregular galaxy II Zw 40 coincides well with
Fig. 5. Relationship between the $[\mathrm{Ne}$ III]/[Ne II $]$ line ratio and the metallicity indicator $\mathrm{Ne} / \mathrm{H}$ for the combined sample of H II regions. Symbols are as in Fig. 1. The data collected on starburst galaxies (open stars) are compared to the Galactic and LMC/SMC H II regions. The parallel lines delineate the parameter space span up by the data. A typical error bar for $\log ([\mathrm{Ne} \mathrm{III}] /[\mathrm{Ne} \mathrm{II}])$ is shown in the left bottom corner. No error bar for $\mathrm{Ne} / \mathrm{H}$ is indicated as the main uncertainty (which can be as large as a factor of 2 for the Galactic sources, see Sect. 5) comes from the abundance derivation method itself and is very hard to determine. The solid lines show the results of the photoionization models calculated by Giveon et al. (2001) using Pauldrach SEDs for subsolar, solar and supersolar stellar and nebular metallicities.

Table 3. [Ne III] /[Ne II] line ratios and $\mathrm{Ne} / \mathrm{H}$ abundances for a sample of galaxies, including $\mathrm{Sgr} \mathrm{A}^{*}$. The sample is sorted out by increasing degree of ionization.

\begin{tabular}{lccc}
\hline \hline $\begin{array}{c}\text { Object } \\
\text { name }\end{array}$ & {$[\mathrm{NeIII}] /[\mathrm{NeII}]$} & $\begin{array}{c}\mathrm{Ne} / \mathrm{H}^{\dagger} \\
\left(10^{-4}\right)\end{array}$ & Ref. \\
\hline M 83 & 0.05 & 2.1 & $1,+$ \\
Sgr A $^{*}$ & 0.06 & 1.8 & $2,+$ \\
NGC 7552 & 0.08 & 1.8 & $1,+$ \\
NGC 253 & 0.08 & 1.8 & $1,+$ \\
NGC 4945 & 0.09 & 1.3 & 3 \\
M82 & 0.18 & 1.4 & 4 \\
NGC 3256 & 0.20 & 2.3 & 5 \\
NGC 4038/39 & 0.92 & 0.6 & 6 \\
NGC 5253 & 4.0 & 0.7 & 7 \\
IIZW 40 & 12 & 0.4 & $1,+$ \\
\hline
\end{tabular}

$\left({ }^{\dagger}\right)$ Calculated from the mid-infrared [NeII] $12.8 \mu \mathrm{m}$, [NeIII] $15.5 \mu \mathrm{m}$ and $\operatorname{Br} \alpha 4.0 \mu \mathrm{m}$ lines assuming $T_{\mathrm{e}}=10^{4} \mathrm{~K}$ and the low density limit. No extinction correction is applied. $\left(^{\ddagger}\right)$ The nature of this galaxy is uncertain. The infrared diagnostics show no evidence for the existence of the AGN inferred from hard X-ray observations (see Spoon et al. 2000). $\left(^{\diamond}\right)$ ISO pointing centered on the overlap region between the two interactinc systems.

References: (1) Thornley et al. (2000); (2) Lutz et al. (1996); (3) Spoon et al. (2000); (4) Förster Schreiber et al. (2001); (5) Rigopoulou et al. (1996); (6) Kunze et al. (1996); (7) Crowther et al. (1999); (+) ISO archive.

the $\mathrm{H}$ II regions in the LMC, further reinforcing our interpretation that metallicity and degree of ionization are strongly interconnected. Among the low ionized galaxies are M 82 and NGC 253, which are the prototypical starburst galaxies. NGC 3256, with a [Ne III]/[Ne II] similar to that of $\mathrm{M} 82$, is a recent galaxy merger. The highly ionized galaxies include NGC 5253, a young, Wolf Rayet galaxy, 
and NGC 4038/4039 (the Antennae), where an extensive burst of star formation is ongoing in the overlap region between the two merger systems.

The $[\mathrm{Ne} \mathrm{III}] /[\mathrm{Ne} \mathrm{II}]$ line ratio and other ionization ratios observed in starburst galaxies have been used to infer the spectral type of the ionizing stars and hence, the age of the starburst (e.g. Schaerer \& Stasińska 1999; Crowther et al. 1999; Thornley et al. 2000; Spoon et al. 2000). However, as this discussion has emphasized, the stellar type is only one of the parameters influencing the ionization structure. Furthermore, the uncertain spectral classification of the ionizing sources through ionization line ratios makes a proper age estimate of the starburst difficult. The other factor influencing the ionization structure, metallicity, measures the cumulative effect of star formation over the galaxy's history.

\section{Summary and conclusions}

In this paper, we have examined the interplay between the ionization structure of H II regions, the SEDs of their ionizing stars and metallicity. To this end, we combined the near- and mid-infrared ISO spectra of a set of Galactic and Magellanic Cloud H II regions. This wavelength range gives us access to the $[\mathrm{S} \mathrm{IV}] 10.5 /[\mathrm{S} \mathrm{III}] 18.7 \mu \mathrm{m}$ and [Ne III] $15.5 /[\mathrm{Ne}$ II] $12.8 \mu \mathrm{m}$ line ratios, which are sensitive to the ionization structure of the nebula. The $[\mathrm{S} \mathrm{IV}] /[\mathrm{S} \mathrm{III}]$ and $[\mathrm{Ne} \mathrm{III}] /[\mathrm{Ne} \mathrm{II}]$ line ratios are found to span a range of more than 2 orders of magnitude and to be highly correlated. The low metallicity LMC and SMC HII regions overlap the trend found for the Galactic objects at the high ionization end.

The dependence of the $[\mathrm{S} \mathrm{IV}] /[\mathrm{S} \mathrm{III}]$ and [NeIII]/[NeII] line ratios on the $T_{\text {eff }}$ of the ionizing star is investigated using a grid of photoionization models at solar metallicity. The models are calculated using two different stellar atmosphere models: the CoStar SEDs from Schaerer \& de Koter (1997) and the more recent SEDs from Pauldrach et al. (2001). The interpretation of the observed ionization line ratios in terms of the stellar content of the $\mathrm{H}$ II region depends critically on the adopted SED. In particular, we note that the CoStar SEDs predict systematically much cooler stars than the Pauldrach SEDs for the same ionization conditions. As a result, practically the whole observed ionization range can be fit by a very narrow $T_{\text {eff }}$ range between $\sim 33000$ and $42000 \mathrm{~K}$ when the CoStar models are adopted, while the entire range up to $55000 \mathrm{~K}$ is suitable when using the Pauldrach models. These differences originate principally from the different treatment of the line blanketing/blocking and wind properties.

For a few sources, spectral types of the ionizing sources are presently available from infrared ( $K$-band) or optical stellar spectroscopy. The comparison of these spectral types with those derived indirectly from the ionized gas through the $[\mathrm{SIV}] /[\mathrm{SIII}]$ and $[\mathrm{NeIII}] /[\mathrm{NeII}]$ line ratios confirms that metallicity has an important influence on the stellar spectra. As impetus for this, we have investigated the relation between the ionization structure and the metallicity comparing the observed [Ne III]/[Ne II] line ratios and $\mathrm{Ne} / \mathrm{H}$ abundances. Although a large scatter is present, a loose correlation between the two parameters is found. This observed trend is compared to photoionization models calculated varying self-consistently the nebular and stellar metallicity (Giveon et al. 2001). The comparison shows that the observed increase in degree of ionization can be explained by the hardening of the SED due to a decrease of the metallicity.

We note, moreover, that the $T_{\text {eff }}$ calibration of the different spectral types is based on modelling the observed photospheric hydrogen, helium and metal lines. This calibration is uncertain as it is very sensitive to the input physics of the stellar model used. The widely used calibration of early stars by Vacca et al. (1996) is based on plane-parallel, non-LTE models which do not incorporate stellar winds and line blanketing. It has been shown, for instance, that the inclusion of line blanketing, stellar wind and a spherical geometry in the models (Martins et al. 2002) lowers the predicted $T_{\text {eff }}$ substantially when compared to the calibrated by Vacca et al. (1996) for the same spectral type. A new calibration is needed if predictions of the stellar content of H II regions are to be compared with the observed spectral types of the ionizing stars. Such a recalibration will have to include the effects of metallicity.

In view of this analysis, the main conclusions are the following:

- The interpretation of the observed $X^{+i+1} / X^{+i}$ ratios is valid only when the stellar metallicity of the SED used in the modelling properly matches the metallicity of the local ISM. Not taking into account the effect of the stellar metallicity correctly can lead to wrong conclusions on the stellar content of the nebula and thus on the local stellar population.

- The adequate treatment of the effect of stellar wind and line blocking and blanketing on the ionizing spectra of early $\mathrm{O}$ stars, and the availability of grids of stellar models at different metallicities, is needed. In this respect, the new models by Pauldrach et al. (2001) constitute an improvement over previous ones.

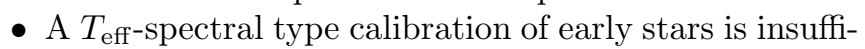
cient if the effects of metallicity are not accounted for. A detailed new calibration is, therefore, necessary.

- The determination of starburst ages in starburst galaxies based on $X^{+i+1} / X^{+i}$ ratios is likely incorrect if metallicity is not taken into account.

Concluding, it is important to further study the ionization structure of $\mathrm{H}$ II regions, particularly using different tracers such as the He I recombination lines. Furthermore, from a theoretical point of view, it may be important to investigate the origin of the actual spectral classification, such as the $\mathrm{K}$ band spectral classification via the C IV and N III photospherical lines and their dependence on the stellar metallicity. 
Acknowledgements. We thank the anonymous referee, whose comments greatly improved this article. MICE is supported at MPE by DLR (DARA) under grants 50 QI 86108 and 50 QI 94023.

\section{References}

Afflerbach, A., Churchwell, E., Acord, J. M., et al. 1996, ApJS, 106, 423

Crowther, P. A., Beck, S. C., Willis, A. J., et al. 1999, MNRAS, 304,654

de Graauw, T., Haser, L. N., Beintema, D. A., et al. 1996, A\&A, 315, L49

Deharveng, L., Peña, M., Caplan, J., \& Costero, R. 2000, MNRAS, 311, 329

Förster Schreiber, N. M., Genzel, R., Lutz, D., Kunze, D., \& Sternberg, A. 2001, ApJ, 552, 544

Ferland, G. J., Korista, K. T., Verner, D. A., et al. 1998, PASP, 110,761

Giveon, U., Sternberg, A., Lutz, D., Feuchtgruber, H., \& Pauldrach, A. 2001, ApJ, 556, 880

Hanson, M. M., Conti, P. S., \& Rieke, M. J. 1996, ApJS, 107, 281

Henry, R. B. C., \& Worthey, G. 1999, PASP, 111, 919

Hillier, D. J., \& Miller, D. L. 1998, ApJ, 496, 407

Kaper, L., Bik, A., Hanson, M., \& Comerón, F. 2002a, in Hot Star Workshop III: The Earliest Stages of Massive Star Birth, ed. P. A. Crowther, ASP Conf. Ser., 267, 95

Kaper, L., Bik, A., Hanson, M., \& Comerón, F. 2002b, in ESO workshop on The Origins of Stars and Planets: The VLT view, ed. J. Alves, \& M. McCaughrean, ESO Astr. Symp., in press

Kessler, M. F., Steinz, J. A., Anderegg, M. E., et al. 1996, A\&A, 315, L27

Kunze, D., Rigopoulou, D., Lutz, D., et al. 1996, A\&A, 315, L101

Lutz, D., Feuchtgruber, H., Genzel, R., et al. 1996, A\&A, 315, L269

Martín-Hernández, N. L., Peeters, E., Morisset, C., et al. 2002, A\&A, 381, 606 (MHP02)
Martins, F., Schaerer, D., \& Hiller, D. J. 2002, A\&A, 382, 999

Massey, P., \& Hunter, D. A. 1998, ApJ, 493, 180

Massey, P., Parker, J. W., \& Garmany, C. D. 1989, AJ, 98, 1305

Morisset, C., Schaerer, D., Martín-Hernández, N. L., et al. 2002, A\&A, 386, 558

Oey, M. S., Dopita, M. A., Shields, J. C., \& Smith, R. C. 2000, ApJS, 128, 511

Pauldrach, A. W. A., Hoffmann, T. L., \& Lennon, M. 2001, A\&A, 375, 161

Peeters, E., Martín-Hernández, N. L., Damour, F., et al. 2002, A\&A, 381, 571

Rigopoulou, D., Lutz, D., Genzel, R., et al. 1996, A\&A, 315, L125

Rolleston, W. R. J., Smartt, S. J., Dufton, P. L., \& Ryans, R. S. I. 2000, A\&A, 363, 537

Schaerer, D., \& de Koter, A. 1997, A\&A, 322, 598

Schaerer, D., \& Stasińska, G. 1999, A\&A, 345, L17

Shaver, P. A., McGee, R. X., Newton, L. M., Danks, A. C., \& Pottasch, S. R. 1983, MNRAS, 204, 53

Simpson, J. P., Witteborn, F. C., Price, S. D., \& Cohen, M. 1998, ApJ, 508, 268

Spoon, H. W. W., Koornneef, J., Moorwood, A. F. M., Lutz, D., \& Tielens, A. G. G. M. 2000, A\&A, 357, 898

Takahashi, H., Matsuhara, H., Watarai, H., \& Matsumoto, T. 2000, ApJ, 541, 779

Thornley, M. D., Schreiber, N. M. F., Lutz, D., et al. 2000, ApJ, 539, 641

Vacca, W. D., Garmany, C. D., \& Shull, J. M. 1996, ApJ, 460, 914

Vermeij, R., \& van der Hulst, J. M. 2002, A\&A, submitted

Vermeij, R., van der Hulst, J. M., Damour, F., \& Baluteau, J.-P. 2002, A\&A, in press

Vílchez, J. M., \& Pagel, B. E. J. 1988, MNRAS, 231, 257

Walborn, N. R., \& Blades, J. C. 1997, ApJS, 112, 457

Watarai, H., Matsuhara, H., Takahashi, H., \& Matsumoto, T. 1998, ApJ, 507, 263

Watson, A. M., \& Hanson, M. M. 1997, ApJ, 490, L165 